\title{
Prevención de adherencias pélvicas postquirúgicas. Revisión de tema
}

\author{
Beatriz Elena Palacio Marín*
}

Recibido: Julio $6 / 2000$

Revisado: Julio 14 / 2000

Aceptado: Agosto $11 / 2000$

\section{RESUMEN}

Cada día se profundiza más sobre el estudio de diferentes de métodos que puedan contribuir a disminuir la aparición de adherencias postquirúrgicas, ya que estas se asocian a gran morbilidad y a altos costos desde el punto de vista económico.

Teniendo en cuenta que la cirugía ginecológica es una de las principales causas de formación de adherencias, el objetivo del presente artículo es presentar una revisión de la literatura analizando la fisiopatología de la formación de adherencias y de los diferentes métodos que han sido utilizados y de los que se están estudiando para su prevención.

PALABRAS CLAVES: Adherencias postquirúrgicas, adhesiolisis, prevención.

\section{SUMMARY}

Every day we know more about the different methods that lower the incidence of postsurgical pelvic adhesions, since those are associated with a high morbidity and costs.

Realizing that gynecological surgery is one of the main causes in the genesis of adhesions, the objective of the present paper is to review of literature concerning the formation of adhesions' phisiology and the different methods used to prevent them.

KEY WORDS: Postsurgical adhesions, adhesiolysis, prevention.

\section{Introducción}

Los esfuerzos hacia la prevención de las adherencias pélvicas se han ido haciendo cada día mayores debido a la gran morbilidad y a los altos costos que conllevan. En Estados Unidos durante 1988 se invirtieron \$254.9 millones de dólares en hospitalización de pacientes que consultaron por dolor pélvico y requirieron adhesiolisis para su tratamiento, además se invirtieron $\$ 925$ millones de dólares en honorarios médicos, para un total de \$1.179.9 millones de dólares, sin tener en cuenta las pérdidas debido a la incapacidad laboral que de ello se generó (1). Teniendo en cuenta que la cirugía pélvica ginecológica es uno de los principales predisponentes para la formación de adherencias, el objetivo de este artículo es hacer una revisión de los eventos fisiopatológicos que llevan a su formación, de los métodos utilizados para su prevención y de lo que se vislumbra hacia el futuro en este aspecto.

\section{Discusión}

Las adherencias peritoneales son bandas fibróticas anómalas de diverso grado de rigidez que conectan superficies de tejidos que normalmente se encuentran separados en la cavidad peritoneal (2). Se encargan de localizar la reacción inflamatoria cuando hay infección o cuerpo extraño y transportan oxígeno al tejido isquémico en caso de fallas en la técnica quirúrgica de una anastomosis intestinal (3).

La edad de las pacientes que consultan por bridas sintomáticas varía entre los 25 y los 50 años y es más frecuente en mujeres de raza negra. Pueden presentarse hasta en un $97 \%$ de las pacientes a quienes se les ha realizado cirugía pélvica, de estas, un 3\% a un $6 \%$ requerirán manejo quirúrgico por obstrucción o dolor. De las pacientes a las que se les realiza adhesiolisis por dolor, un $60 \%$ a un $90 \%$ presentan mejoría sintomática, pero posteriormente reaparecen síntomas atribuibles a éstas en un $30 \%$ de los casos. (4) Los principales problemas asociados a adherencias pélvicas postquirúrgicas son el dolor pélvico crónico $(20 \%$ a $50 \%)$, la obstrucción intestinal $(49 \%$ a $74 \%)$ y la infertilidad (15\% a $20 \%)$. (1) La mortalidad relacionada a las bridas sintomáticas varía entre un $6 \%$ a un $11 \%$ y se le atribuye a las complicaciones secundarias a la obstrucción intestinal, a su tratamiento y al procedimiento de adhesiolisis como tal. (2) La principal causa de adherencias es la cirugía previa $(70 \%$ a $95 \%)$, siendo las cirugías más implicadas las realizadas por peritonitis generalizada, adhesiolisis, endometriosis y tumores ginecológicos. $5 \%$ a $9 \%$ de las bridas son de origen congénito y menos del $1 \%$ son idiopáticas (4).

* Ginecóloga Obstetra. Profesora del Departamento de Ginecología y Obstetricia. Universidad de Antioquia. Medellín. 
Ray y colaboradores encontraron 303.836 hospitalizaciones por procedimientos relacionados a adhesiolisis durante 1993, un $1 \%$ del total de hospitalizaciones en Estados Unidos. El promedio de días estancia fue de 9.7 y el costo promedio por paciente fue de $\$ 22.000$ dólares. El costo de las cirugías realizadas por infertilidad asociada a bridas fue de \$166.9 millones de dólares durante el mismo año. En 1994 el costo total de los procedimientos relacionados a adhesiolisis fue de 1.3 billones de dólares (5).

Para prevenir la formación de adherencias peritoneales es importante tener una adecuada técnica quirúrgica, manipular cuidadosamente los tejidos, realizar una hemostasia selectiva, utilizar suturas adecuadas preferiblemente de tipo sintéticas y absorbibles, no emplear un tiempo quirúrgico mayor de 120 minutos, no usar gasas en el secado, ni aun húmedas, no suturar el peritoneo y evitar el residuo de talco en los guantes quirúrgicos. Sin embargo, aunque se tomen en cuenta todas estas precauciones no es posible prevenirlas en todos los casos. Actualmente se usa polvo de harina en vez de polvo de talco para los guantes, medida que ha demostrado disminuir la incidencia de adherencias (6).

El peritoneo de manera característica comienza a regenerarse mediante siembras de aglomerados de células mesoteliales en el área denudada. La multiplicación y migración de las células mesoteliales de los márgenes de la herida contribuyen al proceso de regeneración, pero no son el evento principal. Figura 1. El tiempo que toma el proceso de regeneración del mesotelio varía entre 3 y 8 días y está relacionado con el área de la lesión. (7) Inicialmente se presenta una reacción inflamatoria con el consiguiente depósito de fibrina, exudado inflamatorio y neovascularización, luego hay una invasión de fibroblastos y factores del crecimiento, formación de colágeno y maduración del mismo y por último la aparición de adherencias fibrosas. (8) Figura 2.

Figura 0018i01

\section{RECUBRIMIENTO PERITONEAL DEL AREA DENUDADA. FLECHA LLENA: SUPERFICIE PERITONEAL. FLECHA ABIERTA: BORDE DE LESIÓN DE PERITONEO. CABEZA DE FLECHA: ISLOTES DE CÉLULAS MESOTELIALES}

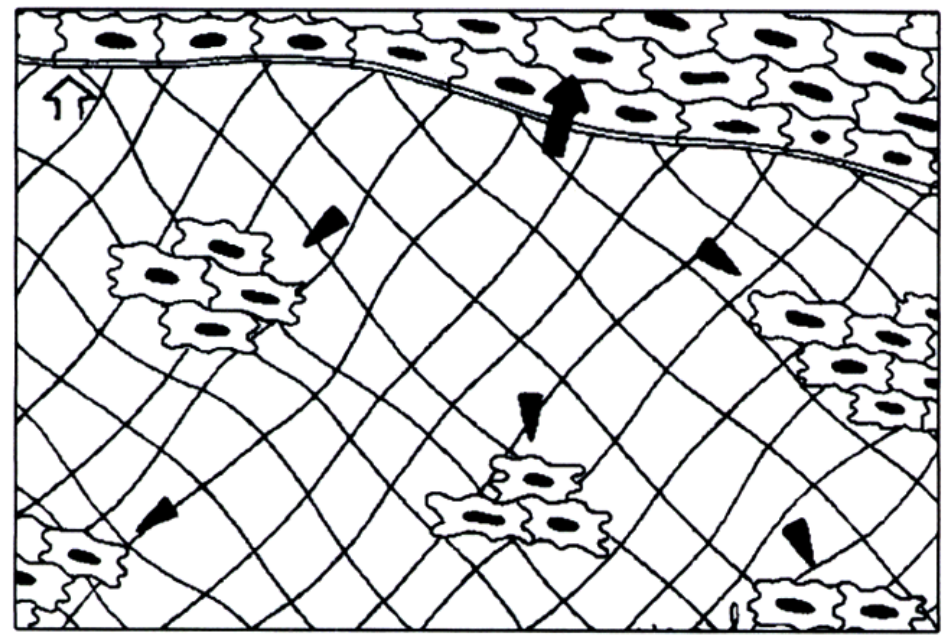

Figura $0018 \mathrm{i} 02$

FISIOPATOLOGIA DE LA FORMACION DE ADHERENCIAS

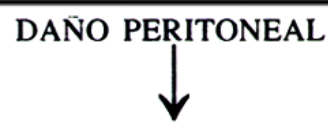

REACCIÓN INFLAMATORIA<smiles>C1CC1</smiles>

DEPÓSITO DE FIBRINA<smiles>C1CC1</smiles>

NEOVASCULARIZACIÓN

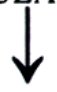

FIBROBLASTOS Y FACTORES DEL CRECIMIENTO<smiles>C1CCCC1</smiles>

COLÁGENO<smiles>C1CC1</smiles>

ADHERENCIAS

El evento etiológico es la injuria peritoneal, lo que genera una respuesta cicatricial que conduce a la formación de bridas. Dicha injuria puede deberse a un trauma quirúrgico, trastornos inflamatorios, procesos infecciosos, infiltración neoplásica, isquemia o irradiaciones. La fibrina se acumula en el sitio de la lesión y favorece la adherencia de otras estructuras cubiertas por peritoneo. La lisis de los depósitos de fibrina es una propiedad fisiológica de las superficies mesoteliales normales. La fibrina se lisa por acción de la plasmina. El Activador del Plasminógeno, liberado por células mesoteliales, convierte plasminógeno en plasmina y esta lisa la fibrina convirtiéndola en productos que son luego absorbidos por el peritoneo. Durante el reparo mesotelial la acción del Activador del Plasminógeno es inhibida por la producción de citoquinas en la superficie peritoneal, lo que determina la formación de adherencias fibrinosas iniciales. Si la inhibición de la actividad fibrinolítica es prolongada, se pueden formar adherencias fibrosas permanentes. La disminución de la actividad del Activador del Plasminógeno comienza en las primeras 6 a 12 horas posteriores a la injuria y su recuperación toma 3 o 4 días, llevando luego a la lisis de la fibrina y a una cicatrización libre de bridas. Cuando se reactiva dicha enzima, la actividad fibrinolítica del peritoneo regenerado aumenta. Es así como el control del sistema fibinolítico de las superficies mesoteliales es un factor importante en la prevención fisiológica de la formación de adherencias (9).

La pérdida de la capa superficial de fosfolípidos del peritoneo puede hacer parte de la patogénesis de las 
adherencias, ya que actúa como lubricante entre las supeficies previniendo su formación, además, el uso de fosfatidilcolina ha demostrado experimentalmente disminuir la aparición de ellas (10).

Soluciones cristaloides tipo Lactato Ringer o Solución Salina se han utilizado para evitar la formación de adherencias postquirúrgicas, sin embargo, los estudios han lanzado resultados contradictorios (11). Los cristaloides se absorben de la cavidad peritoneal a una velocidad de $35 \mathrm{ml} /$ hora lo que indica que si se instilan $200 \mathrm{mI}$, que es el volumen habitualmente utilizado, sería absorbido en 6 horas y el proceso de reepitelialización se demora mucho más que esto.

Los antiinflamatorios tanto esteroideos como no esteroideos limitan la respuesta inflamatoria al trauma en el sitio quirúrgico disminuyendo la formación del exudado fibrinoso. Los resultados de estudios experimentales con el uso de corticosteroides han sido bastante desalentadores, además de sus propiedades farmacológicas, la inmunosupresión y el retardo en la cicatrización limitan su utilización postquirúrgica. Cohen y colaboradores (11) encontraron una disminución significativa en la formación de adherencias utilizando soluciones intraperitoneales que contenían esteroides, sin embargo otros investigadores no han replicado estos hallazgos. Querleu y colaboradores (12) han informado beneficios utilizando dexametasona en pacientes a las que se les realizó microcirugía por infertilidad y a las cuales se les hizo laparoscopia de segunda mirada 3 - 6 meses después. La razón por la cual los esteroides no han comprobado ser benéficos de manera certera pudiera ser que el proceso inflamatorio inicial que se presenta con el trauma peritoneal simplemente sobrepasa la respuesta terapéutica a las dosis que han sido empleados, pero si se aumentan sus dosis, se aumentan a la vez sus efectos indeseables.

Los antiinflamatorios no esteroideos actúan alterando el metabolismo del ácido araquidónico, la actividad de la ciclooxigenasa y la formación de sus productos terminales: tromboxanos y prostaglandinas. Golan y colaboradores (13-14) encontraron que la adición de prostaglandinas F2 alfa y E2 a la cavidad peritoneal de ratas aumentaban la formación de adherencias en el sitio del trauma. En varios modelos animales se demostró el efecto benéfico de los antiinflamatorios administrados por vía sistémica (oxifenbutazona e ibuprofeno) en la prevención de su formación, sin embargo no todos los estudios han logrado demostrar lo mismo. Otros han encontrado que la administración intraperitoneal de antiinflamatorios puede disminuir la formación de adherencias, el problema es que no se ha hallado hasta el momento el vehículo adecuado para su liberación y en áreas desvascularizadas e hipóxicas, que es donde la formación de bridas es mayor, los antiinflamatorios administrados de manera sistémica no llegan (15).

El Dextrán es un polímero de glucosa hidrosoluble que se ha usado para la prevención de formación de adherencias a una concentración del $32 \%$ y con un peso molecular de 70.000 daltons. Este es absorbido de la cavidad peritoneal en 5 a 7 días. En algunos estudios experimentales en animales se encontraron efectos benéficos, (16) pero en otros no. (17) En un estudio en pacientes sometidas a tratamiento microquirúrgico por infertilidad y las cuales fueron evaluadas por laparoscopia de segunda mirada 8 a 12 semanas más tarde, se encontró que la instilación de 250cc de Dextrán en la cavidad peritoneal disminuye la formación de adherencias postquirúrgicas. (18) Rosenberg y Board (19) realizaron un estudio aleatorizado en dos grupos de pacientes que fueron llevadas a cirugía por infertilidad. El primer grupo recibió Dextrán (200cc) y el segundo grupo Lactato Ringer. Se les realizó una segunda laparoscopia a las 12 semanas y demostraron que el segundo grupo presentaba mayor número de adherencias postquirúrgicas con una diferencia estadísticamente significativa. En Suiza se realizó un estudio multicéntrico prospectivo aleatorizado, donde se instiló intraperitonealmente a un grupo $250 \mathrm{mI}$ de dextrán y al otro $250 \mathrm{mI}$ de solución salina al $0.9 \%$, una laparoscopia de control realizada 4 a 10 semanas después, no logró demostrar diferencias entre ambos grupos. El edema de vulva se presenta en un $2 \%$ de las pacientes asociado al uso de dextrán, el cual se resuelve espontáneamente en 4 semanas. Otros efectos secundarios menos frecuentes son la ganancia de peso transitoria, ascitis, edema de miembros inferiores, derrame pleural, cuagulopatía y elevación de transaminasas séricas, todos estos se resuelven espontáneamente o con terapia de soporte. El shock anafiláctico puede ocurrir en un porcentaje mínimo de pacientes $(0.008 \%)(15)$.

Dentro de los métodos de barreras mecánicas que se han utilizado para la prevención de adherencias se encuentran la celulosa oxidada regenerada, la membrana bioabsorbible de hialuronato de sodio y carboximetilcelulosa, y las láminas de politetrafluoretileno expandido (PTFE-e). La barrera física ideal debe ser no reactiva, durar mientras se dan todas las etapas de regeneración mesotelial y luego reabsorberse. Las dos primeras cumplen todos estos criterios y la primera tiene actividad antibacteriana in-vivo (20).

El Interceed, al igual que el Surgicel es celulosa oxidada regenerada, pero con una duración intraperitoneal más prolongada que mantiene su posición sin necesidad de suturas, siempre y cuando haya escaso líquido peritoneal y adecuada hemostasia. La membrana toma la textura de una gelatina y cubre el peritoneo lesionado durante la regeneración mesotelial. Luego esta sustancia es hidrolizada y los productos de su degradación (glucosa y ácido glucurónico) son aclarados de la cavidad peritoneal. El producto se absorbe entre 10 a 14 días y no interfiere con la ovulación. En un estudio experimental en conejos se demostró una disminución en la formación de adherencias intraperitoneales con Interceed. En otro estudio clínico multicéntrico prospectivo aleatorizado se evaluó la eficacia del Interceed. A las pacientes del grupo de infertilidad que fueron llevadas a adhesiolisis, se les colocó Interceed en el área sin peritoneo a un lado de la pared pélvica (lado estudio) y al otro lado no se les colocó nada (lado control). Se realizó una segunda laparoseopia entre 10 días y 14 semanas posteriores a la laparotomía. El lado estudio mostró una disminución significativa en la incidencia, extensión y severidad de las adherencias. Se encontró prevención de adherencias en un $51 \%$ de las 
pacientes a las que se les aplicó Interceed y en un $24 \%$ en el grupo control. El $90 \%$ de las pacientes tratadas con Interceed se beneficiaron de la terapia. Se ha comprobado que cubrir el ovario con la membrana es útil después de procedimientos tales corno cistectomías, perforación ovárica (drilling), resección de endometriomas y ooforoplastias. Estudios en animales han demostrado que el uso de Surgicel reduce la severidad y la incidencia de adherencias postquirúrgicas. Sin embargo otros estudios no han demostrado este beneficio (21-22).

El Seprafilm es una membrana bioabsorbible compuesta por hialuronato de sodio y carboximetilcelulosa. La membrana se hidrata y se convierte en un gel en las primeras 24 a 48 horas después de aplicada y se va absorbiendo lentamente en la primera semana. Todos los productos de su degradación se excretan en un promedio de 28 días. Esta se coloca cubriendo el área de lesión peritoneal y separa las superficies opuestas durante el proceso de cicatrización. Se ha demostrado que el Seprafilm disminuye la incidencia, extensión y severidad de las adherencias postquirúrgicas en la cavidad abdominopélvica. Su seguridad y eficacia han sido demostradas en dos estudios multicéntricos doble ciego aleatorizados y en cuatro ensayos clínicos que involucraron 342 pacientes y además en numerosos estudios en animales. Hasta el momento no se conocen contraindicaciones para su utilización. No se han identificado reacciones de cuerpo extraño, aún no ha sido evaluada en cirugía oncológica ni durante el embarazo (9).

Las membranas de politetrafluoretileno expandido (PTFE-e) son láminas menores de $1 \mathrm{~mm}$ de espesor con poros de menos de $1 \mu \mathrm{m}$ de diámetro que retardan la penetración celular. En cirugía cardiovascular han demostrado su eficacia en la prevención de adherencias cuando se usa como sustituto pericárdico. Sin embargo, los resultados de los estudios en pacientes ginecológicas han sido contradictorios. Estas deben ser fijadas con suturas y no son absorbibles. En procedimientos tales corno enfermedad adherencial severa, áreas de daño peritoneal extenso y resección de leiomiomas, se ha encontrado que el PTFE-e es eficaz para prevenir la formación de adherencias. Sin embargo, en cirugías ginecológicas radicales no se ha demostrado su beneficio (23-24).

La laparoscopia disminuye la formación de nuevas adherencias, pero la formación de las que ya existían sigue siendo un problema mayor. DiZerega encontró en laparoscopias posteriores a una adhesiolisis laparoscópica, que hasta el $67 \%$ de las pacientes tienen adherencias en el sitio donde habían sido lisadas, además, el $16 \%$ de las pacientes presentaron nuevas adherencias. (11) Durante el procedimiento laparoscópico se han utilizado barreras mecánicas (celulosa oxidada regenerada) encontrando que su uso es fácil y se requiere en promedio dos minutos y medio para la aplicación de cada trozo de membrana, utilizando en promedio tres trozos por paciente, lo que significativamente disminuye la reaparición de adherencias (25).

La aproximación del peritoneo por medio de suturas para cubrir las áreas denudadas puede dificultar el reparo peritoneal e incluso aumentar la formación de adherencias. (4) Aun en cirugías radicales pélvicas por cáncer ginecológico se ha demostrado que la aproximación peritoneal es adecuada sin suturarlo y la formación de bridas postquirúrgicas es menor. (26) Tulandi y colaboradores demostraron que no había diferencia en la formación de adherencias postquirúrgicas entre pacientes a las que se les había aproximado el peritoneo posterior a una cesárea con material de sutura y entre las que no. Esta conducta no aumentó el índice de complicaciones y disminuyó el tiempo quirúrgico (27).

El uso de las barreras mecánicas disminuye significativamente la formación de adherencias peritoneales, siempre y cuando sean colocadas adecuadamente, toda el área denudada quede cubierta por ellas, haya adecuada hemostasia y poca cantidad de líquido peritoneal.

\section{Conclusión}

A pesar de todos los estudios realizados y de los avances alcanzados en la investigación sobre la prevención de adherencias pélvicas, todavía permanecen una serie de preguntas sin contestar: ¿Por qué unas pacientes forman adherencias y otras no? ¿Cuál es la diferencia entre reformación y neoformación de adherencias? ¿Por qué existen diferencias potenciales entre la formación de adherencias en diversos tipos de cirugías?

Para encontrar el método ideal para disminuir al máximo la formación de adherencias es importante permanecer en la investigación en esta área y tratar de dar respuesta a estos interrogantes.

\section{BIBLIOGRAFIA}

1. Ray NF. Larsen JW. Stillman RJ, Jacobs RJ. Economic impact of hospitalizations for lower abdominal adhesiolysis in the United States in 1988. Surg Gynecol Obstet 1993; 176: 271-6.

2. Koveker GB. Coerper S, Gottwald T. et al. The role of wound healing in the formation of peritoneal adhesions. In Treutner KH, Schumpelick V (Eds). Peritoneal adhesions. Berlin, Springer Verlag. 1997; 22-28.

3. Hunt TK. Can adhesions be prevented? J Am Coll Surg 1996; 183: $297-$ 306.

4. Moreno A. Adherencias peritoneales posquirúrgicas. Rev Col Cir. 1998; 14: 185-95.

5. Ray NF, Denton WG, Thamer M, et al. J Am ColI Surg 1998; 186: 1-9.
6. Luijendijk RW, DeLand DCD. Wauters CC, et al: Foreign materials in postoperative adhesions. Ann Surg 1996; 223: 242-8.

7. Menzies D. Aetio-pathogenesis of peritoneal adhesions with respect to post-traumatic fibrinolitic activity. In Treutner KH, Schumpelick V (Eds). Peritoneal adhesions. Springer Verlag, Berlin. 1997; 105-110.

8. Miligan DW, Raftery AT. Observations on the pathogenesis of peritoneal adhesions: a light and electron microscopical study. Br. J Surg. 1974; 61: 274-80.

9. www.genzyme.com. 1999.

10. Snioj M, Ar Rajab A, Ahren B, Bengmark S. Effect of phosphatidylcoline on postoperative adhesions after smaIl bowel anastomosis in the rat. Br J Surg. 1992; 79: 427-9. 
11. Cohen BM, Heyman T, Mast D. Use of intraperitoneal solutions for preventing pelvic adhesions in the rat. J Reprod Med 1983; 28: 649-53.

12. Querleu D, Vankeerberghen DF, Deffense F. Boutteville C. The effect of noxytiolin and systemic corticosteroids in infetility surgery: a prospective randomized study. J Gynecol Obstet Biol Reprod. 1989; 18: 935-40.

13. Golan A. Prostaglandins-a role in adhesion formation. An experimental study. Acta Obstet Gynecol Scand 1990; 69: 339-41.

14. Golan A. The effect of prostaglandins and aspirin-an inhibitor of prostaglandin synthesis-on adhesion formation in rats. Hum Reprod 1991; 6: 251-4.

15. DiZerega. G S. Contemporary Adhesion prevention. Fertil Steril. 1994; 60: 219-235.

16. Dlugi AM, DeCherney AH. Prevention of postoperative adhesion formation. Semin Reprod Endocrinol. 1984; 2: 125-29.

17. Vemer HM, Boeckx W, Brosens I. Use of dextrans for the prevention of postoperative peritubal adhesions in rabbits. Br J Obstet Gyneacol. 1982; 89: 473-5.

18. Adhesion Study Group. Reduction of postoperative pelvic adhesions wigh intraperitoneal $32 \%$ dextran 70 : a prospective, randomized clinical trial. Fertil Steril. 1983; 40: 612-9.

19. Rosemberg SM, Board JA. High-molecular weight dextran in human infertility surgery. Am J Obstet Gynecol. 1984; 148: 380-5.
20. Queralt CB, Laguens G, Lozano R, Morandeira JR. Prevention of peritonitis with oxidized regenerated cellulose. Infect Surg 1987; 12: $659-60$.

21. Interceed (TC7) Adhesion Barrier Study Group. Prevention of postsurgical adhesions by INTERCEED (TC7), an absorbable adhesion barrier: a prospective, randolnized rnulticenter clinical study. Fertil Steril 1989; 51: 933-8.

22. Interceed (TC7) Adhesion Barrier Study Group n. Pelvic sidewall adhesion reformation: rnicrosurgery alone or with Interceed absorbable adhesion barrier. Surg Gynecol Obstet 1993; 177: 135-9.

23. Boyers SP, Diamond MP, DeCherney AH. Reduction of postoperative pelvic adhesions in the rabbit with Gore - Tex Surgical Membrane. Fertil Steril 1988; 49: 1066-70.

24. Goldeberg JM, Toledo AA, Mitchell PE. An evaluation of the Gore Tex Surgical Membrane for the prevention of postoperative peritoneal adhesions. Obstet Gynecol 1987; 70: 846-8.

25. Azziz R. Use of an oxidized, regenerated cellulose absorbable adhesion barrier at laparoscopy. J Reprod Med 1991; 36: 479-82.

26. Hubbard TB, Khan MZ, Carag VR, Albites VE, Hricko GM. The pathology of peritoneal repair: its relation to the formation of adhesions. Ann Surg 1967; 165: 908-16.

27. Tulandi T, Hum GS, Gelfand MM. Closure of laparotomy incisions with or without peritoneal suturing and second look laparoscopy. Am J Obstet Gynecol. 1988; 158: 536-7. 